\title{
Clinical Judgement or Implicit Bias: Ethical Challenges of Oesophageal Foreign Body in a Prisoner
}

\author{
Emma Watts ${ }^{1}$, Emma Richards ${ }^{1}$, Nicola Wooles ${ }^{1}$, and Anita Sonsale ${ }^{1}$ \\ ${ }^{1}$ Queen Elizabeth Hospital Birmingham
}

June 8,2020

\section{LETTER TO THE EDITOR:}

A 28-year-old prisoner presented with a one-year history of dysphagia and weight loss (BMI 18). Initially, he self-discharged from another trust after CT neck suggested a plastic foreign body encapsulating softer material lodged within a pharyngeal pouch at the level of T2-T3 (Figure 1). Referred as having "?Swallowed drugs", he denied ingesting any non-food items or recreational drugs although remained unable to provide a cohesive history. Despite recently undergoing investigation for mental health concerns, he reported no other significant past medical history. Sinus bradycardia (33bpm) with a normal QTC incited suspicion of systemic absorption of intra-corporeal illicit drugs. Given the inconsistent history, bradycardia and imaging, the team maintained high suspicion and pre-emptively sought guidance on the ethics of drug testing and preservation of evidence. Unexpectedly, a plastic bottle cap was removed during rigid panendoscopy, which contained digested food substances. Pharyngoscopy revealed a significant oesophageal stricture at $24 \mathrm{~cm}$ with no cricopharyngeal bar suspicious of chronic inflammatory stricture. 24-Hour ambulatory ECG recording confirmed only sinus bradycardia. He was discharged back to prison once eating and drinking, aiming for recurrent dilatation every two weeks until luminal diameter reached $>15 \mathrm{~mm}$.

Foreign body ingestion remains rare in adults but prevalence is greater in the elderly, drug traffickers, prison inmates and patients with psychiatric conditions[1]. Intentional ingestion of foreign bodies is more common in incarcerated populations due to perceived secondary gain, pica or psychiatric conditions[2]. Malingerers display repetitive, intentional ingestion of multiple items and may be associated with other self-harm behaviours[3]. Objects typically include sharp metals, batteries or plastic-wrapped illegal drugs. The prevalence of drug abuse within prison populations is high, with illicit substances often concealed by body packing[4]. Rupture of packaging with consequent leakage of contents may result in lethal intoxication. Imaging is notoriously difficult to interpret as scybala, fruit stones or grains may imitate packaged drugs.

Clinical judgement remains pivotal in formulating diagnoses and optimising medical management. However, balancing rational associations with unconscious bias is ethically challenging. A cautious balance must be struck between implicit bias and clinical suspicion when considering the management of oesophageal foreign bodies. Overall, we propose it is clinically safer to manage such patients under the presumption that any packaging contains illicit substances. However, no patient should have their care compromised due to race, gender, age or background. As prison populations continue to rise, further work needs to be done to tackle implicit bias within a healthcare setting.

WORD COUNT: 398 words

\section{REFERENCES:}

1. - Ribas Y, Ruiz-Luna D, Garrido M, Bargallo J, Campillo F. Ingested foreign bodies: do we need a specific approach when treating inmates? Am Sure 2014 Feb;80(2):131-7. 
2. - Evans DC, Wojda TR, Jones CD, Otey AJ, Stawicki SP. Intentional ingesions of foreign objects among prisoners: A review. World J Gastrointest Endosc. 2015;7(3):162-168.

3. - Volpi A, Laforgia R, Lobito C, Panebianco A, Punzo C, Ialongo P et al. Ingestion of foreign bodies among prisoners: a ten years retrospective study at the University Hospital of Southern Italy. G Chir. 2017 Mar-Apr;38(2):80-83.

4. - Traub SJ, Hoffman RS, Nelson LS. Body Packing: the internal concealment of illicit drugs. NEJM 2003;349:2519-26.

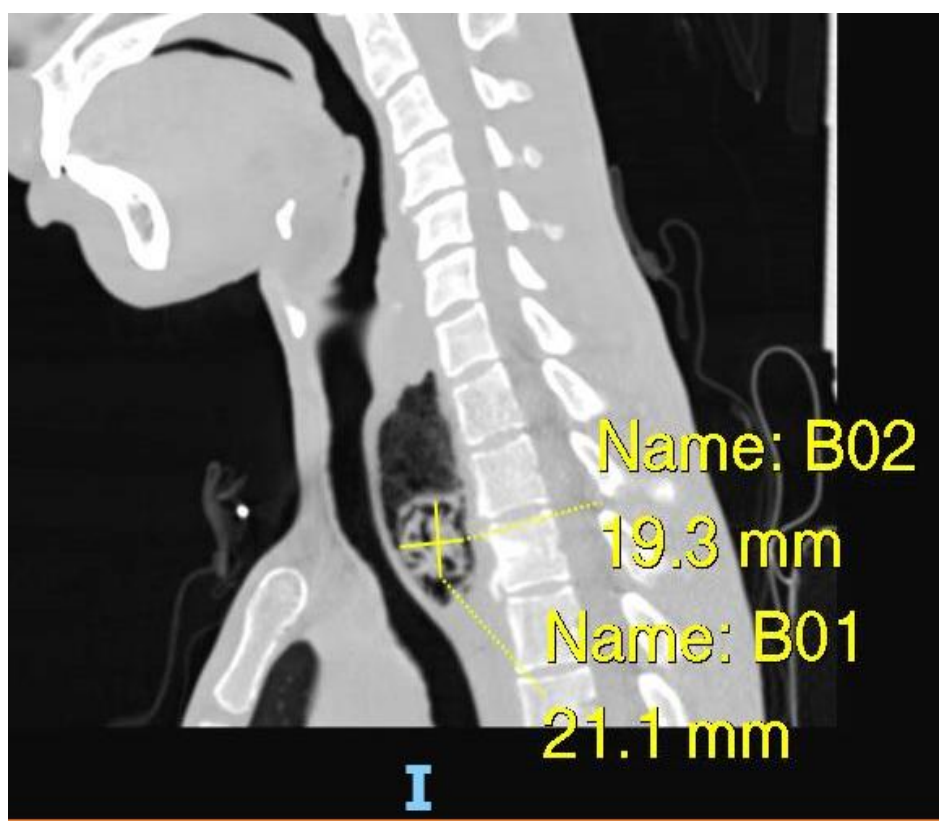

